\title{
Thoracic CT-PET Registration Using a 3D Breathing Model
}

\author{
Antonio Moreno ${ }^{1}$, Sylvie Chambon ${ }^{1}$, Anand P. Santhanam ${ }^{2,3}$, \\ Roberta Brocardo ${ }^{1}$, Patrick Kupelian ${ }^{3}$, Jannick P. Rolland ${ }^{2}$, Elsa Angelini ${ }^{1}$, \\ and Isabelle Bloch ${ }^{1}$ \\ ${ }^{1}$ Ecole Nationale Supérieure des Télécommunications (GET - Télécom Paris), \\ CNRS UMR 5141 LTCI - Signal and Image Processing Department, Paris, France \\ ${ }^{2}$ Optical Diagnostics and Applications Laboratory, University of Central Florida, \\ USA \\ ${ }^{3}$ Department of Radiation Oncology, MD Anderson Cancer Center Orlando, USA
}

\begin{abstract}
In the context of thoracic CT-PET volume registration, we present a novel method to incorporate a breathing model in a non-linear registration procedure, guaranteeing physiologically plausible deformations. The approach also accounts for the rigid motions of lung tumors during breathing. We performed a set of registration experiments on one healthy and four pathological data sets. Initial results demonstrate the interest of this method to significantly improve the accuracy of multimodal volume registration for diagnosis and radiotherapy applications.
\end{abstract}

\section{Introduction}

Registration of multimodal medical images is a widely addressed topic in many different domains, in particular for oncology and radiotherapy applications. We consider Computed Tomography (CT) and Positron Emission Tomography (PET) in thoracic regions, which provide complementary information about the anatomy and the metabolism of the human body (Fig. 1). Their registration has a significant impact on improving medical decisions for diagnosis and therapy 1223 . Linear registration is not sufficient to cope with local deformations produced by respiration. Even with combined PET/CT scanners which avoid differences in patient orientation and provide linearly registered images, nonlinear registration remains necessary to compensate for cardiac and respiratory motions [4].

Most of the existing non-linear registration methods are based on image information and do not take into account any knowledge of the physiology of the human body. Landmark-based registration techniques do take physiology into account by forcing homologous points to match. In this direction, several breathing models were built for medical visualization, for correcting artefacts in images or for estimating lung motion for radiotherapy applications, but few papers exploit such models in a registration process (Section 21).

In this paper, we propose to integrate a physiologically driven breathing model into a 3D non-linear registration (Sections 3 and 4). The registration problem is defined between two CT volumes and one PET volume (Fig. 11). 
(a)

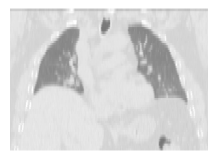

(b)

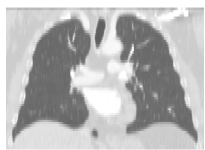

(c)

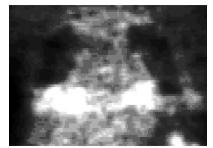

Fig. 1. CT images (a,b) corresponding to two different instants of the breathing cycle and PET image (c) of the same patient (coronal views)

\section{Breathing Models}

Breathing Models and Thoracic Imaging Registration - Currently, respirationgated radiotherapies are being developed to improve the efficiency of radiations of lung or abdominal tumors [5]. Three techniques have been proposed so far: (i) active techniques controling the patient's breathing (airflow is blocked); (ii) passive or empirical techniques using external measurements in order to adapt radiation protocols to the tumor's motion 6678; ; (iii) model-based techniques employing a breathing model to evaluate lungs deformations during the breathing cycle 9]. Different bio-mathematical representations of the human respiratory mechanics have been developed 10. Among Mathematical tools, the most popular technique is based on Non-Uniform Rational B-Spline (NURBS), surfaces which are bidirectional parametric representations of an object. In [11, NURBS surfaces were used to correct for respiratory artifacts of SPECT images, building NCAT (NURBS-based cardiac-torso) model. A multi-resolution registration approach for 4D Magnetic Resonance Imaging (MRI) was proposed in [12] with NCAT. In 13, a 4D NCAT phantom and an original CT image were used to generate 4D CT and to compute an elastic registration. Physically-based models, describing the important role of airflow inside the lungs, can be based on Active Breathing Coordinator (ABC allows clinicians to pause the patient's breathing at a precise lung volume) 9] or on volume preservation relations [14 15]. In [16], segmented MRI data were used to simulate PET volumes at different instants of the breathing cycle. These estimated PET volumes were used to evaluate different PET/MRI registration processes. Authors of [1217] used pre-register MRI to estimate a breathing model. CT registration using a breathing model was presented in [9] but a specific equipement is needed. From a modeling and simulation point of view, physically-based deformation methods are better adapted for simulating lung dynamics and are easy to adapt to the patient, without the need for physical external adaptations.

Physics-Based Dynamic 3D Surface Lung Model - We employ an approach that was previously discussed in [15] and in which the two major components involved in the modeling efforts include: (1) Parameterization of PV (Pressure Volume) data from a human subject which acts as an ABC; (2) Estimation of the deformation operator from 4D CT lung data sets. In step (1) a parameterized PV curve, obtained from a normal human subject, is used as a driver for simulating the 3D lung shapes at different lung volumes. In step (2), the computation takes as inputs the nodal displacements of the $3 \mathrm{D}$ lung models and the estimated amount of 
force applied on the nodes of the meshes (which are on the surface of the lungs). Displacements are obtained from 4D CT data of a normal human subject. The direction and magnitude of the lung surface point's displacement are computed using the volume linearity constraint, i.e. the fact that the expansion of lung tissues is linearly related to the increase in lung volume. The amount of applied force on each node (that represents the air-flow inside lungs) is estimated based on a PV curve and the lungs's orientation with respect to the gravity, which controls the air flow. Given these inputs, a physics-based deformation approach based on Green's function (GF) formulation is estimated to deform the 3D lung surface models. Specifically the GF is defined in terms of a physiological factor, the regional alveolar expandability (elastic properties), and a structural factor, the inter-nodal distance of the 3D surface lung model. To compute the coefficients of these two factors, an iterative approach is employed and, at each step, the force applied on a node is shared with its neighboring nodes, based on local normalization of the alveolar expandability, coupled with inter-nodal distance. The process stops when this sharing of the applied force reaches equilibrium. For validation purposes, a 4D CT dataset of a normal human subject with four instances of deformation was considered [18]. The simulated lung deformations matched the 4D CT dataset with $2 \mathrm{~mm}$ average distance error.

\section{Combining Breathing Model and Image Registration}

We have conceived an original algorithm in order to incorporate the breathing model described above in our multimodal image registration procedure. Fig. 2 shows the complete computational workflow. The input consists of one PET volume and two CT volumes of the same patient, corresponding to two different instants of the breathing cycle (end-inspiration and end-expiration, for example, collected with breath-hold maneuver). The preliminary step consists in segmenting the lung surfaces (and, eventually, the tumors) on the PET data and on the two CT data sets, using a robust mathematical-morphology-based approach [19], and extracting meshes corresponding to the segmented objects.

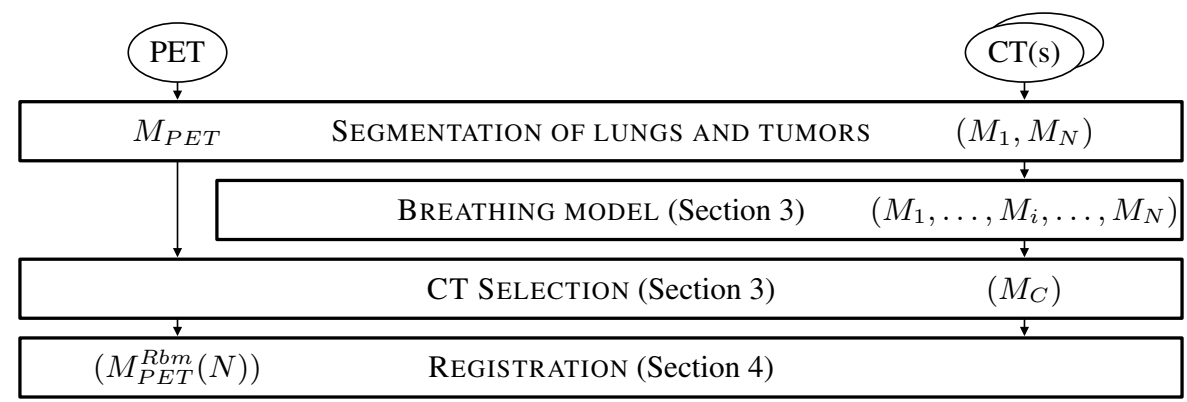

Fig. 2. Registration of CT and PET volumes using a breathing model 
Computation of a Patient-Specific Breathing Model - For each patient, we only have two segmented CT datasets, therefore we first estimate intermediate 3D lung shapes between these two datasets and then, the displacements of lung surface points. Directions are given by the model (computed from a 4D CT normal data set of reference) while magnitudes are "patient-specific" (computed from the given 3D CT lung datasets). With known estimations of applied force and "subject-specific" displacements the coefficients of the GF can be estimated (Section 2). Then, the GF operator is used to compute the 3D lung shapes at different intermediate lung volumes.

$C T$ Selection - Let us denote the CT simulated meshes $M_{1}, M_{2}, \ldots, M_{N}$ with $M_{1}$ corresponding to the $\mathrm{CT}$ in maximum exhalation and $M_{N}$ to maximum inhalation. By using the breathing model, the transformation $\phi_{i, j}$ between two instants $i$ and $j$ of the breathing cycle can be computed as: $M_{j}=\phi_{i, j}\left(M_{i}\right)$. Our main assumption is that even if the PET volume represents an average volume throughout the respiratory cycle, using a breathing model, we can compute a $\mathrm{CT}$ volume that can be closer to the PET volume than the original $\mathrm{CT}$ volumes. By applying the continuous breathing model, we generate simulated CT meshes at different instants ("snapshots") of the breathing cycle. By comparing each CT mesh with the PET mesh $\left(M_{P E T}\right)$, we select the "closest" one (i. e. with the most similar shape). The mesh that minimizes a measure of similarity $C$ (here the root mean square distance) is denoted as $M_{C}: M_{C}=\arg \min _{i} C\left(M_{i}, M_{P E T}\right)$.

Deformation of the PET - Once the appropriate CT $\left(M_{C}\right)$ is selected, we compute the registration, $f^{r}$, between the $M_{P E T}$ mesh and the $M_{C}$ mesh as:

$$
M_{P E T}^{r}(C)=f^{r}\left(M_{P E T}, M_{C}\right),
$$

where $M_{P E T}^{r}(C)$ denotes the registered mesh. Then, the transformation due to the breathing is used to register the PET to the original CT (continuous line in Fig. 3) incorporating the known transformation between $M_{C}$ and $M_{N}$ :

$$
\Phi_{C, N}=\phi_{N-1, N} \circ \ldots \circ \phi_{C+1, C+2} \circ \phi_{C, C+1} .
$$

We apply $\Phi_{C, N}$ to $M_{P E T}^{r}(C)$ in order to compute the registration with $M_{N}$ :

$$
M_{P E T}^{R b m}(N)=\Phi_{C, N}\left(M_{P E T}^{r}\right)=\Phi_{C, N}\left(f^{r}\left(M_{P E T}, M_{C}\right)\right),
$$

where $M_{P E T}^{R b m}(N)$ denotes the PET registered mesh using the breathing model.

A direct registration, denoted $f^{R d}$, can also be computed between $M_{P E T}$ and the original CT mesh $M_{N}$ (dashed line in Fig. [3): $M_{P E T}^{R d}(N)=f^{R d}\left(M_{P E T}, M_{N}\right)$, where $M_{P E T}^{R d}(N)$ is the result of registering the PET directly to the CT mesh $M_{N}$ (note that this could be done with another instant $M_{i}$ ). In the direct approach the deformation itself is not guided by any anatomical knowledge. In addition, if the PET and the original CT are very different, it is likely that this registration procedure will provide physically unrealistic results. 


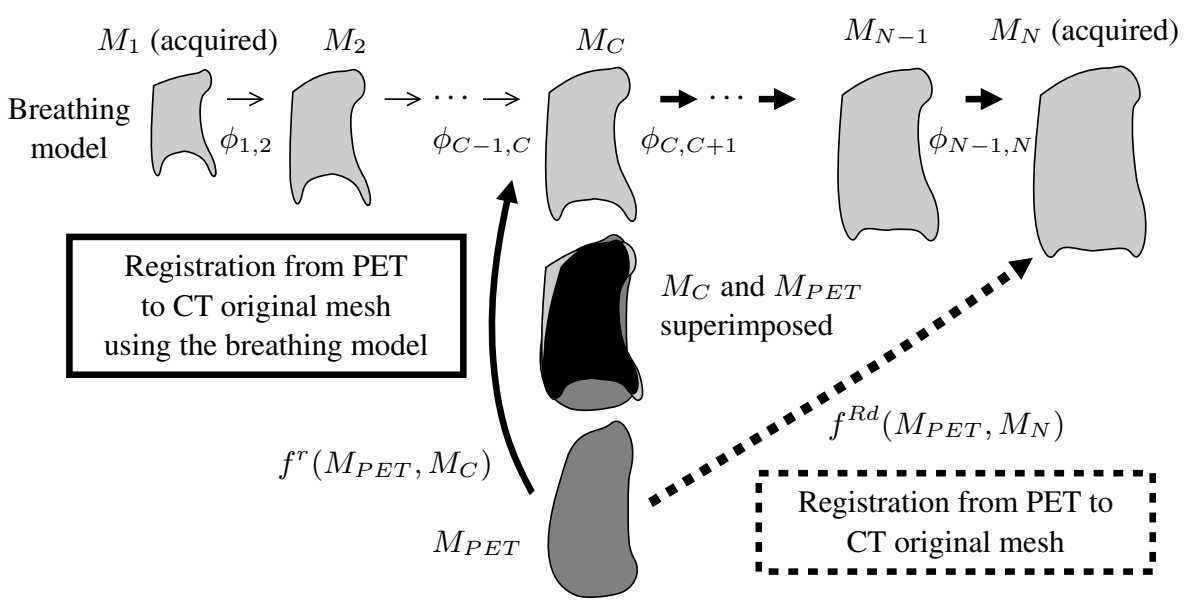

Fig. 3. Registration framework on PET $\left(M_{P E T}\right)$ and CT mesh $\left(M_{N}\right)$ - The $M_{C}$ mesh is the closest to the $M_{P E T}$ mesh. We can register $M_{P E T}$ to the $M_{N}$ mesh (original $\mathrm{CT}$ ) following one of the two paths.

\section{Registration Method Adapted to Pathologies}

The algorithm described in Section 3 can be applied with any type of registration method, to estimate $f^{R d}$ and $f^{r}$. These functions may be computed by any registration method adapted to the problem. We show here how the proposed approach can be adapted for registration of multi-modality images in pathological cases.

Registration with Rigidity Constraints - We have previously developed a registration algorithm for the thoracic region taking into account the presence of tumors, while preserving continuous smooth deformations [20]. We assume that the tumor is rigid and that a linear transformation is sufficient to cope with its displacements between CT and PET scanning. This hypothesis is relevant and in accordance with the clinicians' point of view, since tumors are often compact masses of pathological tissue. The registration algorithm relies on segmented structures (lungs and tumors). Landmark points are defined on both datasets to guide the deformation of the PET volume towards the CT volume. The deformation at each point is computed using an interpolation procedure where the specific type of deformation of each landmark point depends on the structure it belongs to, and is weighted by a distance function, which guarantees continuity of the transformation.

Registration with Rigidity Constraints and Breathing Model - Here, the following procedure is used to compute $f^{r}$ (in our example $M_{N}$ is the original CT):

1. Selection of landmark points on the CT mesh $M_{C}$ (based on Gaussian and mean curvatures and uniformly distributed on the lung surface) [21]; 
2. Estimation of corresponding landmark points on the PET mesh $M_{P E T}$ (using the Iterative Closest Point (ICP) algorithm [22]);

3. Tracking of landmark points from $M_{C}$ to the CT mesh $M_{N}$ using the breathing model;

4. Registration of the PET and the original CT using the estimated correspondences with the method summarized in the previous paragraph.

The breathing model used in step (3) guarantees that the corresponding landmarks selected on the original CT are correct (and actually they represent the same anatomical point) and follow the deformations of the lungs during the respiratory cycle.

\section{Results and Discussion}

We have applied our algorithm on a normal case and on four pathological cases, exhibiting one tumor. In all cases, we have one PET (of size $144 \times 144 \times 230$ with resolution of $4 \times 4 \times 4 \mathrm{~mm}^{3}$ or $168 \times 168 \times 329$ with resolution of $4 \times 4 \times 3 \mathrm{~mm}^{3}$ ) and two CT volumes (of size $256 \times 256 \times 55$ with resolution of $1.42 \times 1.42 \times 5 \mathrm{~mm}^{3}$ to $512 \times 512 \times 138$ with resolution of $0.98 \times 0.98 \times 5 \mathrm{~mm}^{3}$ ), acquired during breathhold in maximum inspiration and in intermediate inspiration, from individual scanners. The breathing model was initialized using the lung meshes from the segmented CT. Ten meshes (corresponding to regularly distributed instants) are generated and compared with the PET. The computation time can reach two hours for the whole process (a few seconds for segmentation, a few minutes for landmark point selection and about ninety minutes for registration). Although this is not a constraint because we do not deal with an on-line process, this computation time will be optimized in the future.

As illustrated in Fig. 4 and 5 (one normal case and one pathological case), the correspondences between landmark points on the original CT and the PET are more realistic in the results obtained with the breathing model (images (e) and (f)) than without (images (b) and (c)). Using the model, it can be observed that the corresponding points represent the same anatomical points and that the uniqueness constraint is respected, leading to visually better looking PET registered images. In particular, the lower part of the two lungs is better registered using the model, the lung contour in the registered PET is closer to the lung contour in the original CT, cf. Fig. 4 $(\mathrm{g}-\mathrm{i})$. In the illustrated pathological case, the tumor is well registered and not deformed. Moreover, the distance between the registered PET lungs and the original CT lungs is lower than using the direct approach.

In this paper, we consider the impact of the physiology on lung surface deformation, based on reference data of normal human subjects. Therefore the methodology presented in this paper will further benefit upon the inclusion of patho-physiology specific data once established. The use of normal lung physiology serves to demonstrate improvements in CT and PET registration using a physics-based 3D breathing lung model. Current work includes a quantitative comparison and evaluation on a larger database, in collaboration with clinicians. 
(a)

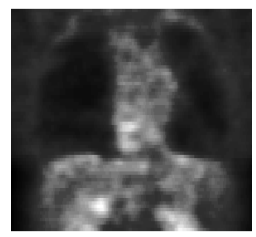

(d)

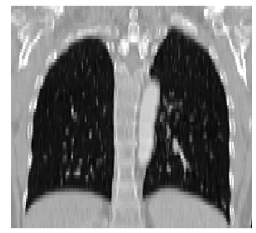

(g)

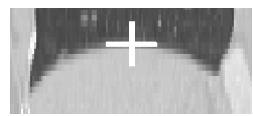

(b)

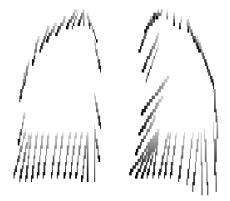

(e)

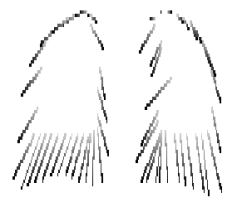

(h)

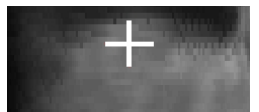

(c)

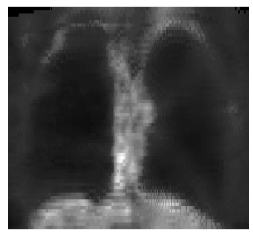

(f)

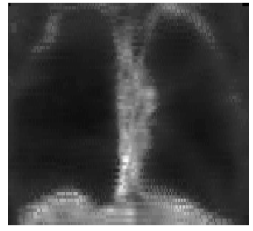

(i)

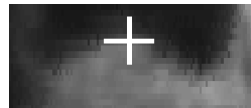

Fig. 4. (a) Original PET, (d) CT images in a normal case. Correspondences between selected points in the PET image and in the CT image are shown in (b) for the direct method and (e) for the method with the breathing model (corresponding points are linked). The registration result is shown in (c) for the direct method and in (f) for the method with the breathing model. Details of registration on the bottom part of right lung, (g) CT, (h) PET registered without breathing model, (c) with breathing model. The white crosses correspond to the same coordinates.

(a)

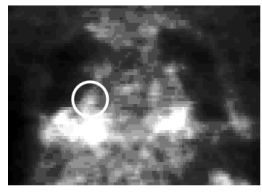

(d)

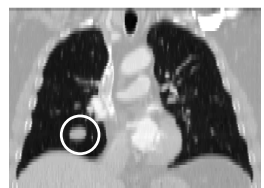

(b)

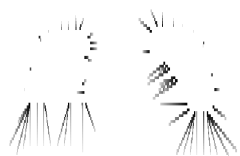

(e)

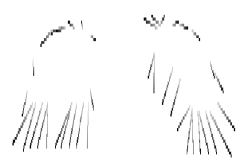

(c)

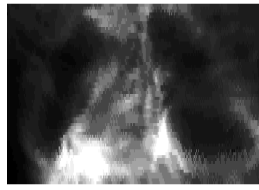

(f)

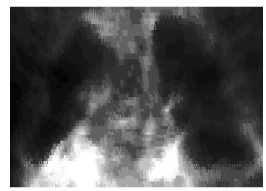

Fig. 5. Same as in Fig. $4(\mathrm{a}-\mathrm{f})$ for a pathological case (the tumor is surrounded by a white circle)

\section{References}

1. Lavely, W., et al.: Phantom validation of coregistration of PET and CT for imageguided radiotherapy. Medical Physics 31(5), 1083-1092 (2004)

2. Rizzo, G., et al.: Automatic registration of PET and CT studies for clinical use in thoracic and abdominal conformal radiotherapy. Physics in Medecine and Biology 49(3), 267-279 (2005)

3. Vogel, W., et al.: Correction of an image size difference between positron emission tomography (PET) and computed tomography (CT) improves image fusion of dedicated PET and CT. Physics in Medecine and Biology 27(6), 515-519 (2006) 
4. Shekhar, R., et al.: Automated 3-Dimensional Elastic Registration of Whole-Body PET and CT from Separate or Combined Scanners. The Journal of Nuclear Medicine 46(9), 1488-1496 (2005)

5. Sarrut, D.: Deformable registration for image-guided radiation therapy. Zeitschrift für Medizinische Physik 13, 285-297 (2006)

6. McClelland, J., et al.: A Continuous 4D Motion Model from Multiple Respiratory Cycles for Use in Lung Radiotherapy. Medical Physics 33(9), 3348-3358 (2006)

7. Nehmeh, S., et al.: Four-dimensional (4D) PET/CT imaging of the thorax. Physics in Medecine and Biology 31(12), 3179-3186 (2004)

8. Wolthaus, J., et al.: Fusion of respiration-correlated PET and CT scans: correlated lung tumour motion in anatomical and functional scans. Physics in Medecine and Biology 50(7), 1569-1583 (2005)

9. Sarrut, D., et al.: Non-rigid registration method to assess reproducibility of breathholding with $\mathrm{ABC}$ in lung cancer. International Journal of Radiation OncologyBiology-Physis 61(2), 594-607 (2005)

10. Mead, J.: Measurement of Inertia of the Lungs at Increased Ambient Pressure. Journal of Applied Physiology 2(1), 208-212 (1956)

11. Segars, W., et al.: Study of the Efficacy of Respiratory Gating in Myocardial SPECT Using the New 4-D NCAT Phantom. IEEE Transactions on Nuclear Science 49(3), 675-679 (2002)

12. Rohlfing, T., et al.: Modeling Liver Motion and Deformation During the Respiratory Cycle Using Intensity-Based Free-Form Registration of Gated MR Images. Medical Physics 31(3), 427-432 (2004)

13. Guerrero, T., et al.: Elastic image mapping for 4-D dose estimation in thoracic radiotherapy. Radiation Protection Dosimetry 115(1-4), 497-502 (2005)

14. Zordan, V., et al.: Breathe Easy: Model and Control of Human Respiration for Computer Animation. Graphical Models 68(2), 113-132 (2006)

15. Santhanam, A.: Modeling, Simulation, and Visualization of 3D Lung Dynamics. $\mathrm{PhD}$ thesis, University of Central Florida (2006)

16. Pollari, M., et al.: Evaluation of cardiac PET-MRI registration methods using a numerical breathing phantom. In: IEEE International Symposium on Biomedical Imaging, ISBI pp. 1447-1450 (2004)

17. Sundaram, T., Gee, J.: Towards a Model of Lung Biomechanics: Pulmonary Kinematics Via Registration of Serial Lung Images. Medical Image Analysis 9(6), 524$537(2005)$

18. Santhanam, A., et al.: Modeling Simulation and Visualization of Real-Time 3D Lung Dynamics. IEEE Transactions on Information Technology in Biomedicine. (in press, 2007)

19. Camara, O., et al.: Explicit Incorporation of Prior Anatomical Information into a Nonrigid Registration of Thoracic and Abdominal CT and 18-FDG WholeBody Emision PET Images. IEEE Transactions on Medical Imaging 26(2), 164-178 (2007)

20. Moreno, A., et al.: Non-linear Registration Between 3D Images Including Rigid Objects: Application to CT and PET Lung Images With Tumors. In: Workshop on Image Registration in Deformable Environments (DEFORM), Edinburgh, UK, pp. 31-40 (2006)

21. Chambon, S., et al.: CT-PET Landmark-based Lung Registration Using a Dynamic Breathing Model. In: International Conference on Image Analysis and Processing, Modena, Italy (2007)

22. Besl, P., McKay, N.: A Method for Registration of 3-D Shapes. IEEE Transactions on Pattern Analysis and Machine Intelligence 14(2), 239-256 (1992) 